280 Bernhard Pollack: Einige Bemerkungen über die Neuroglia etc.

jetzt nach 5 Monaten gut gehalten. Auch dient ein doppeltes Färben zur Vervollkommnung der Resultate ebenso, wie es Weigert schon selbst bei seiner von ihm modificirten Markscheidenfärbung angegreben.

Angesichts des Umstandes aber, dass wir des Thíerexperimentes nicht entrathen können, da wir ja am Menschen keine Versuche mit experimenteller Degeneration anstellen können, erscheint mir die Anwendbarkeit anf das thierische Nervensystem als das nächste Postulat dieser hochbedeutsamen Methode.

Berlin, den 20. Juli 1896.

(Aus dem II. anatomischen Institut zu Berlin.)

\title{
Die interstitiellen Zellen des Hodens und ihre physiologische Bedeutung.
}

$$
\text { Von }
$$

\section{Julius Plato.}

Hierzu Tafel XII.

Angeregt durch ein Präparat vom Testikel des Ebers, welches ich der Güte des Herrn Prosector Dr. Martin Heidenhain, Privatdocenten zu Würzburg, verdankte, entschloss ich mich im Wintersemester 1894-95 zu 'dem.Versuche, die Frage nach der Bedeutung der unter den widersprechendsten Angaben in der Litteratur und den Lehrblichern behandelten sog. Zwischensubstanz mit Hülfe neuerer Methoden der Entscheidung näher zu bringen. - Mit den ersten Vorarbeiten beschäftigt, wurde ich durch äussere Umstände an der Fortsetzung meiner Studien verhindert, die ich erst ein Jahr später, um Weihnachten 95, im II. anatomischen Institute zu Berlin wieder aufnehmen konnte. 
Meiner ursprïnglichen Absicht entsprechend, verschaffte ich mir aus dem hiesigen Centralviehhofe unter allen Vorsichtsmaassregeln Schweineembryonen aus allen Entwicklungsstarlien. Allein so leicht dieses Material zu beschaffen war, so wenig entsprach es den Anforderungen an Frische, die ich in Anbetraclit meines Arbeitsplanes, der mir die Perspektive auf cellularhistologische Studien eroffnete, an dasselbe stellen musste. - So wählte ich denn ein schwieriger zu bescbaffendes, aber genauer kontrollirbares Material, den Testikel des Katers, der hinsichtlich der Mächtigkeit seiner Zwischensubstanz etwa an dritter Stelle in der Reihe der Säuger steht.

Meine Untersuchungen sind nun keineswegs beendigt, und ich möchte den vorliegenden Blättern mehr den Charakter einer ersten Mittheilung, als den einer in allen Theilen abgeschlossenen Arbeit geben. - Bevor ich jedoch an die Mittheilung von Befunden gelie, die einiges Licht in das Dunkel der angeregten Frage zu werfen geeignet $z u$ sein scheinen, sei mir eine kurze Besprechung der einschlägigen Litteratur gestattet.

Entdeckt wurden die interstitiellen Zellen beim Menschen von Koelliker (1), der sie der Gruppe der bindegewebigen Elemente zuzählt.

Selr genau hat sic Henle (2) beschrieben, der sie nicht für bindegewebige Elemente hält, sich jedoch über die Natur oder Bedeutung derselben nicht klar geworden ist.

Einige Jahre später erschien das Lehrbnch I e y di g's (3), der die interstitielle Substanz zum ersten Male zum Gegenstand einer vergleichend-anatomischen Studie machte. Leydig findet bei allen untersuchten Säugethieren im Bindegewebe zwischen den Samenkanälchen neine zellenartige Masse," welche bei einigen Thieren die Samenkanälchen selbst von allen Seiten umgiebt, während sie bei anderen weniger stark entwickelt ist. - Er hält diese Elemente für fett- und pigmenthaltige Bindesubstanzzellen.

Ludwig und Tomsa (4) lassen die Lymphbahnen zwischen den interstitiellen Zellen entstehen und bemerken zur Natur dieser Elemente dann nur ganz kurz: Es ngehen von den Adventitien der Blutgefässe zahlreiche fibrilläre Fortsätze ab . . . die Anschwellungen, welche die Fibrillen zeigen, müssen auf dieselbe Weise wie oben gedeutet werden, als Stücke von schief 
abgeschnittenen Gefässwänden und als eingelagerte zellenartige Körper".

Ebner (5) bespricht ebenfalls die Beziehungen der Zwischensubstanz zu den Blutgefässen beim Hund, dem Kater, dem Kaninchen und dew Menschen. Er beschreibt die Zellen als von unregelmässiger, polygonaler Form, mit scharf conturirtem Kerne und einem deutlichen Kernchen, und namentlich bei alten Individuen sehr pigmentreichem Inhalt. $\mathrm{Er}$ schliesst sich im abrigen der Ansicht Ko elliker's und Ley dig's an, ndass diese Zellengruppen eine besondere Art von Bindegewebe darstellen.

$\mathrm{H}$ of $\mathrm{m}$ e is t e $\mathrm{r}$ (6) spricht von Zellen nepithelialen Charakters", die unabhängig seien von der Richtung der Blutgefässe. Von hohem Interesse sind die entwicklungsgeschichtlichen Angaben dieses Autors, welcher bei einem 4monatlichen Menschenfoetus $2 / 3$ des ganzen Parenchyms von der interstitiellen Substanz. eingenommen fand. Später schwindet dieselbe nach seinen Angaben, bis sie bei einem 8jührigen Knaben nur etwa $1 / 10$ des Volums beträgt, um in der Periode der Geschlechtsreife einc erneute Vermehrung zu erfahren. Beim 24 Jahre alten Manne ist die Zwischensubstan\% stark entwickelt und die Zellen enthalten meist Fett und Pigment. - Das Fett fehlt nach Angabe dieses Autors selten. - Ausserdem glaubt $\mathrm{H}$ o f m e is ter innerhalb ein und derselben Zelle ein antagonistisches Verhalten zwischen Fett und Pigment konstatiren zu können und denkt an genetische Beziehungen zwischen beiden Substanzen. Der Verfasser untersuchte dann noch eine Anzahl Säugethiere und fand „bei zahlreichen Verschiedenheiten in morphologischen Eigenschaften und Anordnung der Zwischenzellen allenthalben übereinstimmende chemische Reaktionen ${ }^{*}$. In keinem funktionirenden Hoden seiner Untersuchungsreihe fehlte das Fett der interstitiellen Zellen. - Bezüglich der Natur dieser Elemente steht Hofmeister auf dem Standpunkte Kölliker's, L e y d i g's und Ebner',s, ja er glaubt sogar alle Uebergangsformen zwischen fixen Bindegewebszellen und typischen interstitiellen Zellen nachweisen zu können. Nur beim Eber und beim Hengst sollen die beiden Gewebsarten unvermittelt nebeneinander bestehen.

Mihalkowicz (7) empfiehlt zu Untersuchungen der Zwischensubstanz den Katerhoden. Er findet dort die Substanz in 
Form von „Drüsengängen" und glaubt, dass wie beim Kater, so bei allen Thieren das eigentlich Charakteristische der Zwischenzellen das Fett sei, welches er selbst noch im Hoden eines ganz ausgehungerten Hundes erhalten findet.

Ganz isolirt mit seiner Ansicht steht $\mathrm{Har}$ wey (8), welcher in den interstitiellen Zellen nervöse Gebilde, nein eigenthümliches, ausserordentlich reichhaltiges Beispiel von mit Zellen versebenen, kernhaltigen vasomotorischen Nervenfasern" sieht.

W a l de y er's (9) Bezeichnung als "Plasmazellen", welche besonders gerne Fett, theils in grossen, theils in kleinen Tropfen aufnehmen, fand dagegen eine allgemeinere Aufnahme in die Lehrbiicher. Jedoch finden wir in einer neueren Arbeit dieses Autors (10) die Erklärung, dass die interstitiellen Zellen wede! mit den Mastzellen Ehrlich's noch mit den Plasmazellen Un na's identisch seien, vielmehr eine besondere Zellenart dar'stellen.

In einer im Jahre 1879 erschienenen ausführlichen Arbeit J a c obs on's (11), der auch die vorstehenden Literaturangaben z. Th. entnommen sind, finden wir dann noch die interessante Angabe, dass bei pathologischen Zuständen beim Hunde die interstitielle Substanz bis zum Verschwinden der Kanälchen vermehrt sei.

Die folgenden Jahre haben unsere Kenntnisse von der Bedeutung der Zwischensubstanz kaum wesentlich bereichert. In einer langen Reilhe gerade in den 80er Jabren erschienener Arbeiten über die Entwicklung des Urogenitalsystems glaubten eine Anzahl von Autoren Anhaltspunkte genug zu finden, den alten widersprechenden Ansichten über die bindegewebige, peritheliale oder gar nervöse Natur besagter Zellen noch eine neue hinzuzufügen, dass nämlich die interstitiellen Zellen nichts anderes seien, als Rudimente eines embryonalen Organs. - Diese Ansicht verdient, wie ich, gestützt auf die Meinung eines neueren Autors (H a n s e mann l. c.) glaube, nur die Bezeichnung einer unwahrscheinlichen Hypothese, selbst rom Standpunkte der damaligen Zeit aus. - Einer Hypothese, da kein Autor diesen Ursprung zu beweisen vermochte, einer unwabrscheinlichen Hypothese, da sich wohl schwerlich ein rudimentäres Organ so innig und gleichmässig in ein funktionirendes einschalten möchte, und ausserdem die nicht widerlegten Angaben $\mathrm{H}$ of m e is ter's über die Ver- 
mehrung der Substanz im funktionirenden Horlen gegenüber dem ruhenden, die Bezeichnung als rudimentäres Organ schon damals als vollständig unhaltbar erselieinen lassen konnte.

Auch Nagels (12) Angabe, dass die interstitiellen Zellen erst bei einem $10 \mathrm{~cm}$ langen menschlichen Embryo auftreten, würde mit obiger Auffassung nicht in Einklang zu bringen sein.

Es schien mir nun im höchsten Grade wahrscheinlich, dass ein Organ, welches in den verschiedenen Entwicklungs- und Funktionsstadien des Hodens ein so verschiedenes Aussehen darbietet, zu der spezifischen Funktion dieser Drüse, der Bildung des Samens, in einer engeren Beziehung stehen müsse, als bisher angenommen wurde, und dass die in der Literatur zerstreut aufzufindenden widersprechenden Angaben über Anlüufung, Aussehen und Inhalt der interstitiellen Zellen bei ein und demselben Thiere eben darauf zurïckzuführen seien, dass den verschiedenen Autoren bald in voller Funktion begriffenc, bald nicht oder wenig funktionirende Hoden vorgelegen haben.

Ich war schon zu bestimmten Resultaten gelangt, als mir zwei nenere Arbeiten uber meinen Gegenstand zugänglich wurden, die mich in meiner Auffiassung nur bestärken konnten.

$\mathrm{H}$ ansemann (13) machte die interessante Entdeckung, dass der Hoden des Murmelthieres bei ruhender Spermatogenese im Zustande des Winterschlafes keine intertistielle Zellen zeigt, während ein sich sehr wild gebärlendes Thier 2 Monate nach Beendigung des Winterschlafes in der thätigen Drüse die interstitielle Substanz in einer an ein grosszelliges Sarkom erinnernden Mächtigkeit aufweist. - Wäbrend die interstitiellen Zellen im funktionierenden Hoden des normalen Meuschen nach den Angaben dieses Autors kaum aufzufinden sein sollen, konstatiert er eine. Vermehrung bei Tuberculose, Krebscachexie und syphilitischer Kachexie, "ohne sonstige Betheiligung des Hodens, " und eine Vermehrung höchsten Grades bei perniciöser Anämie und bei einem Potator. Nach einer längeren Erörterung über die Bedeutung dieser Zellen für die Geschwulstbildung, kommt dẹ Verfasser dann zu dem Schlusse, dass wir in denselben ein bestimmtes Organ mit veränderlicher physiologischer Funktion zu sehen haben.

Reinke (14) untersuchte den Hoden eines 25jährigen Hingerichteten und fand dort - im Gegensatze zu den Angaben 
$\mathrm{H}$ ans c man n's - eine reichlich entwickelte Zwischensubstanz, ähnlich wie sie $\mathrm{Henle}$ (a. a. O.) beschreibt. Der Verfasser stellt mit der Weigert'schen Fibrinfürbung in Alcoholpräparaten ${ }_{n}$ in den interstitiellen Zellen eine grosse Menge von intensiv färbbaren Körpern" dar, "die grosse Aehnlichkeit mit Krystallen haben". Er. bezeichnet sie als neiweissartige Krystalloide", die in allen kräftigen Hoden mit Spermabildung anzutreffen seien, dagegen vermisst werden bei einem 15järigen Jungen und einem 6 b̆jährigen Manne, deren Hoden keine Spermatogenese zeigen. Der Verfasser glaubt, dass dieser Befund "vielleicht in Zukunft im Stande sein wird, auf die richtige Spur zu führen".

Um meinen Untersuchungen keine allzu breite Basis zu geben, beschränkte ich mich vorerst im wesentlichen auf das Studium des funktionirenden Katerhodens und richtete mein Augenmerk vor allem auf den wesentlichen Inbalt der Zwisehenzellen, auf das von allen Autoren übereinstimmender Weise in denselben angetroffene Fett.

Das für meine $Z$ wecke beste Fixationsmittel fand ich in dem Hermann'schen Platin-Osmiumgemisch $(1 \%$ Platinchlorid 15 , $2 \%$ Osmiumsäure 4, Eisessig $1 \mathrm{Th}$.), welches ich in folgender Weise verwende: Dem chloroformirten, noch lebenden Thiere spalte ich das Scrotum, befreie den Hoden von den ihn umgebenden Hüllen bis auf die derbe Albuginea und bis an den Leistenkanal heran, so dass ersterer frei an dem Samenstrange aus der Bauchhöhle heraushängt, lege an der höchsten Stelle eine feste Ligatur um den Samenstrang und injicire darauf mit der Pravazspritze unter mässigem Drucke $1-2 \mathrm{ccm} \mathrm{Hermann}$ 'sche Flüssigkeit schräg unter die Albuginea an einer möglichst tief gelegenen Stelle. - Sofort gewinnt der Hoden eine harte Consistenz. Ich schneide iln darauf dicht unterhalb der Ligatur $a b$, theile ihn mit einem scharfen Rasiermesser in eine obere und eine untere Hälfte und lege diese in nicht zu wenig Hermannsche Flïssigkeit. Dort lasse ich die Stïcke 3 Tage lang und wasche sie dann 24 Stunden in fliessendem Wasser gründlich ans. Hierauf kommen sie in Alcohol von allmählich steigender Concentration. Im 70 oder $80 \%$ Alcohol ziehe ich mit äusserster Vorsicht und so, dass ich das Präparat stets unter Spiritus halte, die Albuginea $a b$, wobei ich mein Augenmerk vorzüglich darauf richte, dass ich einerseits die Hodensubstanz nicht durch Druck 
beschädige - hicrbei leistet mir bei der oberen Hälfte der Funiculus spermaticus, bei der unteren der Kopf des Nebenhodens eine vorzügliche Handhabe - andererseits aber auf der Innenseite der abgezogenen Albuginea möglichst wenig schwarze Punkte oder Stränge erhalte, welche aus durch Osmium geschwärzten interstitiellen Zellen bestehen. - Darauf Weiterbehandlung in Alcohol 95\%, Alcohol abs., Alcohol-Chloroform aa, Chloroform, Chloreform-Paraffin, je 24 Stunden, und schliesslich 1 Stunde in weichem und 2 Stunden in hartem Paraffin. Nun fertige ich von der Durchschnittsfläche in der ganzen Dicke des Hodens $5-10 \mu$ dicke Serienschnitte an, breite sie vorsichtig tiber der Flamme aus und klebe sie mit Eiweiss-Glycerin auf. Endlich löse ich das Paraffin in Xylol und schliesse das Präparat in Xylol-Canadabalsam ein.

Auf diese Weise behandelte Stücke zeigen allerdings vor ilrem Einschlusse in Paraffin an der Durchschnittsfläche eine leichte trichterförmige Einzichung, so dass die ersten - übrigens besten - Schnitte die centralen Theile des Hodens noch nicht treffen, im übrigen jedoch liefert dieses Verfahren ganz vorzügliche Resultate. - Bei der gewöhnlichen Fixationsmethode sind wir nümlich rgenöthigt, zwischen zwei Uebeln zu entscheiden. Fixiren wir. den Hoden in toto, so erhalten wir nur einige Zelllagen unter der Albuginea gut conservirt, schneiden wir den Hoden dagegen in Stücke, so düren wir die auf diese Weise gewonnenen mikroskopischen Bilder nicht obne weiteres auf die Verhältnisse im Leben ubertragen, da durch den Druck der Albuginea beim ersten Einschnitt die eigentliche Drüsensubstanz bervorquillt, und so die einzelnen Theile gegen einander verschoben werden. Mit der Injektionsmethode jedoch erhalten wir fast ganz glatte Schnittflächen, die Dislokation der einzelnen Elemente ist vermieden, und wir gewinnen ein brauchbares Bild aus allen Theilen. Injektionspräparate mit Z en k er'scher Flüssigkeit geben weniger gute, aber immerhin noch ganz brauchbare Resultate.

Betrachten wir nun ein auf die vorstehend angegebene Weise erhaltenes Präparat des Katerhodens bei schwacher Vergrösserung: (Fig. 1), so füllt uns vor allem der Wechsel zwischen ganz dunklen und ganz hellen Parthien auf. Die hellen Parthien entsprechen den Durchschnitten der Tubuli, die dunklen 
bilden bald ein dic Zwischenrïume der Tubuli ausfïllendes Netzi mit verdickten Knotenpunkten, bald legen sie sich in Form von Halbmonden, Strängen oder Nestern der Wandung der Kanälchen dicht an. Sie haben meistens ein feinkörniges Aussehen, jedoch kommen auch Stellen vor, an denen diese Körnchen zu confluiren scheinen. Zuweilen hebt sich von dem schwarzen Untergrunde ein heller Kreis mit einem dunklen Punkte in der Mitte ab. Diese dunkleren Parthien sind wiederum durchzogen von bald mehr bald weniger breiten, helleren Strängen, Gefässen und bindegewebigen Zügen.

Ein ganz anderes Bild bieten die mit Z enker'scher Flussigkeit fixirten und nach der $\mathrm{He}$ id e $\mathrm{nh}$ a in' schen EisenalaunHämatoxylinmethode gefärbten Präparate (Fig. 2). An Stelle der schwarzen Züge finden wir hier Stränge nnd Nester grosser, bald mehr bald weniger gegen einander abgeplatteter und deutlich begrenztè Zellen, mit einem oder mehreren, meistens excentrisch gelegenen runden, bläschenförmigen Kernen. Der Kern selbst zeigt in der Regel einen grossen Nucleolus und von diesem gegen eine peripherische Chromatinschicht radiär ausstrahlende, und in der Oberflaiche des Kernes verlaufende zarte Chromatinbälkchen. Das reichlich vorhandene Protoplasma hat einen typischen Bau. Die Hauptmasse finden wir in der Regel an der dem Centrum der Zelle zugekehrten Seite des randständigen Kernes, und von dieser ausstrahlend durch Querzüge verbundene protoplasmatische Radien. - Das ganze Bild ladet förmlich $\mathrm{zu}$ cytomechanischen Studien im Sinne Martin Heidenhains ein, jedoch gelang es mir trotz eifrigen Suchens nicht, in einem der öfters in der dichtesten Anhäufung des Protoplasmas anzutreffenden Körnchen ein typisches Centrosoma zu entdecken. Auch kam mir bei meinem Objekte niemals eine indirekte Zelltheilung in der Zwischensubstanz zu Gesicht. - Diese Zellen sind bei weitem am zahlreichsten in der interstitiellen Substanz vertreten; es finden sich jedoch nicht gerade selten auch Elemente, welche im grossen und ganzen den eben beschriebenen sehr ähnlich sehen, sich jedoch durch eine geringere Grösse, ein stärker tingirbares und weniger in Maschen angeordnetes Protoplasma und einen mehr central gelegenen Kern auszeichnen, welcher sich nach der Heidenhainschen Methode diffus färbt und nur noch mit Mühe ein dunkler gefürbtes Körnchen als Nucleolus erkennen lässt. Während wir 
bier vielleicht ein früheres Entwicklungsstadium der typischen interstitiellen Zellen vor nns haben, finden wir in geringerer Anzahl noch Elemente, welche in regressiver Metamorphose begriffen zu sein scheinen. Diese Zellen übertreffen an Grösse die zuletzt beschriebenen um das Doppelte und zeigen innerhalb verschwommener Zellgrenzen ein aus dïnnen Bälkchen bestehendes, weitmaschiges Protoplasmanetz mit einer excentrisch gelegenen Protoplasma-Anlıäufung, ohne dentlich nachweisbaren Kern.

BezügTich der Natur der im allgemeinen sehr deutlichen Zellgrenzen branche ich nur auf die Arbeit von L adwig und. To $\mathrm{m}$ s a hinzuweisen, welche zwischen den einzelnen interstitiellen Zellen die Lymphbalınen beginnen lassen. Ich kann für den Katerhoden diesen Befund bestätigen. Mittels Einstichinjektion von chinesischer Tusche unter die Albuginea gelang es mir leicht, diese Lymphspalten an einzelnen Stellen injicirt $\mathrm{zu}$ erhalten. Machte ich die Injection intra vitam and liess das Thier einige Stunden leben, so fand ich auch vereinzelte Tuschekörnchen in den interstitiellen Zellen selbst. Die oben geschilderten, eigenthïmlichen, schwamnartigen Protoplasmastrukturen erregen zunächst den Verdacht, unter dem Einflusse der Z en k e r'schen Flüssigkeit entstandene Kunstprodukte zu sein. Dieser Verdacht ist insofern erklärlich, als wir nur höchst selten mit diesel Flüssigkeit Präparate erhalten, in denen nicht die interstitielle Substanz sowohl in sich selbst zerklifftet, als auch durch weite arteficielle Spalträume von der Wandung der Kanälchen getrennt wäre, was doch in letzter Instanz auf eine Schrumpfung der Elemente zuriickzufübren ist. - Dass aber diese Protoplasmastrukturen nicht unter dem Einflusse der Fixation entstehen, vielmehr den lebenden Zellen in typischer Weise zu eigen sind, das beweisen die Hermann'schen Präparate. Wenn wir hier die durch Osmium hervorgerufene Schwärung durch Behandlung der Schnitte mit Wasserstoffsuperoxyd beseitigen, so finden wir nun an Stelle der schwarzen Züge. und Nester die typischen interstitiellen Zellen, wie sie uns die Z enker-Hei de $\mathrm{nh}$ a i n'schen Bilder zeigen, und in den Protoplasmalïcken nunmehr farblose Körner, die in osmirtem Zustande das oben geschilderte schwarzkörnige Aussehen der interstitiellen Substanz bedingten. - Die erwähnten helleren Kreise auf dunklem Grunde entsprechen den Kernen der beschriebenen kleineren Elemente, und wo die 
schwarzen Körner dort dichter liegen und fast $\boldsymbol{z}$ konfluiren scheinen, finden wir hier die grossen Zellen mit dem weitmaschigen Protoplasmanetz und den verschwommenen Zellgrenzen.

Die Frage nach der chemischen Constitution der eingelagerten Körner will ich hier nicht erörtern, und obgleich ich eine genaue chemische Analyse derselben für sehr wünschenswerth halte, sie, den Angaben der älteren Literatur folgend, als Fettkörner bezeichnen. Eine Darstellung derselben auf die von $R$ einke (a. a. 0.) angegebene Weise ist mir nicht gelungen und möchte ich sie also auch nicht für identisch erklüren mit den von diesem Autor beschriebenen Krystalloiden.

Wir sahen, dass die Fettkörner im funktionirenden Hoden des Katers in einer solchen Menge auftreten, dass sie in osmirtem Zustande sowohl den Bau der einzelnen Zellen, als die Struktur der ganzen Zwischensubstanz verdecken. - Fügen wir noch hinzu, dass das Verhältniss beim noch nicht funktionirenden Hoden, sowie bei alten Thieren ein anderes ist, so erscheint die Vermuthung gerechtfertigt, dass wir über die Bedeutung der Zellen nicht mehr im unklaren wären, wofern wir nur über die V.erwendung ihres wesentlichen Inhaltes etwas Näheres wüssten.

Form, Grösse und Intensität der Schwärzung mit Osmium schwanken bei den Fettkörnern innerhalb weiter Grenzen. Die runde Grundform tritt selten rein in die Erscheinung, da die Körner sich in ihrer grossen Masse in einen verhältnissmässig engen Raum theilen müssen. Am meisten finden wir innerhalb der Zellen rund ovale oder gegen einander abgeplattete, oder aber an einer oder mehreren Seiten mit buckelförmigen Hervortreibungen versehene Formen. Von diesen bis zu den eckigen und langgestreckten finden sich alle Uebergänge. Auch eingeschnürte Körner kommen vor, gehören aber zu den Seltenheiten.

Die Körner können in der Grösse der grossen oben beschriebenen Zellen auftreten, in der Regel aber erreichen sie nicht einmal die Grösse der Zellkerne und kommen schliesslich auch in der Grösse der Nucleolen ror.

Die Intensität der Schwärzung mit Osmium hat die verschiedensten Grade. Vom tiefsten Deckschwarz bis zur leichtesten Bräunung finden sich alle Schattirungen. Selbst die einzelnen Körner sind zuweilen an einer Seite dunkler als an der andern. Auffallend ist ferner, dass bei der allmählichen Entfärbung, der 
diese Präparate leider alle anheimfallen, einzelne Körner bevorzugt werden, während andere in unmittelbarer Nähe noch lange Zeit illre tiefe Farbe bellalten.

Suchen wir uns nun über die Bezielıungen der interstitiellen Substanz zu den Drïsentubuli klar zu werden. - Die Tubuli erscheinen auf dem Querschnitte in der Regel von doppelten Contouren begrenzt, an welche sich nach aussen unmittelbar die interstitiellen Zellen mit ihrem reichen Fettgehalte anlegen. Betrachten wir die Grenzcontouren unter starker Vergrösserung, so sehen wir, dass die Wandung der Tubuli keine in sich geschlossene Membran ist, als welche sie den älteren Autoren imponirte, dass sie vielmehr Lücken und Spalten aufweist, welche das Innere der Tubuli mit den interstitiellen Räumen verbinden. Ja man kann in Anbetracht der oft nicht unbetrïchtlichen Dicke der aus mehreren Lagen von Bindegewebsfibrillen gebildeten Wandungen, wie ich glaube von „Kanälchen" reden, und werde ich diese meines Wissens bisher noch nicht in ihrer Bedentung erkannten Gebilde fortan mit diesem Namen hier bezeichnen.

In Fig. 4 a sehen wir cine besonders schöne Stelle. Bei $\mathrm{k}$ sind die rechts und links gauz scharfen Contouren der Wandung eines Tubulus in einer Breite, welche ungefähr dem Durchmesser eines Kernes der interstitiellen Zellen entspricht, unterbrochen, und wir finden dort eine helle Masse mit Andeutungen einer gegen das Lumen der Tubuli convergirenden Streifung. Die seitliche Begrenzung dieses Kanälchens wird durch zwei scharfe, im selben Sime convergirende Linien gebildet, welche sich bis tief in die anliegenden interstitiellen Zellen hinein rerfolgen lassen, und in dem ron ihnen begrenzten, im Innern des Tubulus gelegenen verjüngten Raume ein abgestumpft keilförmiges, mit seitlichen Einschurungen versehenes Fettkorn enthalten. - Kurz und gut, wir haben hier einen sich aus der interstiellen Substanz in das Innere des Tubulus senkenden Trichter vor uns, der die Wandung des Tubulus durchbohrt, und in dessen breiter Oeffnung und engerer Mündung sich Fetttropfen finden. Der Trichter befindet sich hier an einer ganz typischen Stelle, in dem Fusse einer Sertolischen Zelle. Es beweist dies sowohl die an das innere Fettkorn sich gegen das Lumen des Tubulus anschliessende parallelstreifige Substan , als vor allem die Betrachtung des nächsten Schnittes der Serie, der in Fig. 4 b dargestellt ist. Hier 
sieht man an der entsprechenden Stelle nichts mehr von einem Kanälchen, das die Wandung des Tubulus durchbohrt, und nur die etwas helleren Contouren der letzteren denten darauf bin, dass sie hier vielleicht nicht in der ganzen Dicke des Schnittes vorhanden ist. An Stelle des grossen keilförmigen Fetttropfens sehen wir hier den breiten Fuss der Sertolischen Zelle, und in demselben einige kleine Fetttröpfchen, deren Form und Anordnung darauf hinweisen, dass sie ursprünglich mit dem grossen Fetttropfen im vorigen Sclinitte zusammen hingen und durch das Messer von demselben abgetrennt worden sind. Die interstitielle Substanz ist hier in etwas grösserer Ansdehnung getroffen und die Lage des grossen bläschenförmigen Kernes beweist die obige Behauptung, dass der die Wandung durchbohrende Trichter in der interstitiellen Zelle selbst und nicht etwa in den Saftlücken der interstitiellen Substanz oder sonstwo seinen Ursprung nimmt.

Ich suchte nun in meinen Präparaten nach ähnlichen Befunden und entdeckte an einer ganzen Reihe entsprechender Stellen in der Wandung der Tubuli Lücken, die stets zu in der Nähe liegenden Fettkörnchen Beziehungen zu haben schienen. Bald lagen diese Fetttropfen dicht vor den Kanälchen in den interstitiellen Zellen, bald lagen sie im Tubulus am anderen Ende des Kanälehens, in den meisten Fällen jedoch fand ich sie noch ganz oder theilweise in dem Kanälchen selbst (Fig. 7).

Haben wir es hier nicht mit Kunstprodukten zu thun? Von den vielen und griindlichen Autoren, welche bisher mit denselben Reagentien und Hülfsmitteln dasselbe Objekt, wenn auch $\mathrm{zu}$ anderen Zwecken, bearbeiteten, hat keiner auf derartige Befunde aufmerksam gemacht oder sie gar abgebildet, es sei denn, dass mir die diesbezïglichen Angaben entgangen wären, was ich jedoch nicht glaube. Von keiner Driise ferner sind analoge Thatsachen bekannt. - Und trotzdem glaube ich fulr die Richtigkeit meiner Deutung der Präparate mit voller Sicherheit eintreten zu können, denn ich halte es für nicht wohl denkbar, dass das Messer, sei es auf dem Hin- oder'Rückwege ein Fettkorn aus den interstitiellen Zellen herausheben, in eine typische Form bringen und unter scharf begrenzter Verletzung der Wandung der Tubuli gerade in den Fuss der Sertolischen Zelle niederlegen sollte, ohne dass auch die nächstliegenden Parthien desselben Schnittes und die entsprechenden Parthien an den nächsten 
Schnitten derselben Seric dureh dicselbe Stelle des Messers in ähnlicher Weise beschädigt werden müssten. Auch müsste man den Fettkörnern dann eine Unnachgiebigkeit gegen das Messer, ja eine fast elastische Consistenz zuschreiben, dic sie gewiss nicht besitzen, und schliesslich miissten die Fettkörnchen in ihrer Lage von der Schnittrichtung bestimmt werden. Von alle dem ist nichts zu bemerken. - Eine zweite Gelegenheit zur Bildung von Kunstprodukten bietet das Aufkleben der Schnitte. Es kommt in der That bei diesem Objekte trotz der grössten Sorgfalt bei der Bchandlung nicht selten vor, dass sich gerale die Randpartbien der Tubuli mit der anstossenden interstitiellen Substanz in einer schmalen Falte umlegen oder über einander schieben und so zu verkehrten Beobachtungen Anlass geben könnten. Allein derartige Stellen sind leicht an den die Dicke des Schnittes übertreffenden Niveaudifferenzen der einzelnen Körner und Zellenbestandtheile zu erkennen, und ich habe es mir zur Pflicht gemacht, in jedem einzelnen Falle erst mit den stärksten Ocularen und mit ausge\%ogenem Tubus auf zu grosse Niveaudifferenzen zu fahnden, bevor ich die betreffende Stelle als beweisend anerkenne.

Nach Ausschaltung der Möglichlieit, dass wir es mit Kunstprodukten $z$ thun haben, fragt es sich nun, ob durch diese Kanäle eine Strömung von Fettkörnchen stattfindet, und wenn dies der Fall ist, in welcher Richtung. - Für die Entscheidung der ersten Frage fehlt mir leider eine exakte Mcthode, allein ich glaube nicht, dass man mir den Vorwurf allzu kiulner Schlüsse wird machen können, wenn ich aus der Thatsache, dass wir die Fettkörnchen hald ror, bald ganz oder theilweise in den Kanälchen finden, eine Bejahung dieser Frage abstrahire.

Da eine Reibe von Injektions-Versuchen, die ich behufs Entscheidung der zweiten Frage, nach der Richtung der Fettkörnchenströmung anstellte, keine absolut beweisenden Resultate ergab, so suchte ich uber diese Verhältnisse mit Hülfe der Entwicklungsgeschichte und der vergleichenden Anatomie Aufklärung zu erlangen.

Die Entwicklungsgeschichte des Katerhodens, auf die ich in einer späteren Arbeit ausführlicher einzugehen gedenke, lehrte mich vor allem die Thatsache, dass die Fettkörnchen zuerst in den interstitiellen Zellen, und zwar bereits in einem sehr frühen 
Stadium auftreten, in welchem die letzteren noch durch breite bindegewebige Lamellen von der Wandung der Tubuli getrennt sind, und eine direkte Communication zwischen diesen und den interstitiellen Zellen nicht nachweisbar ist. Auch findet man in den Tubuli selbst zu dieser Zeit keine Fetttropfen. Mit zunehmendem Wachsthum des Hodens legen sich dann die interstitiellen Zellen immer enger an die Wand der Tubuli an. Die Entwicklungsgeschichte der interstitiellen Substanz spricht also für eine aus derselben gegen das Lumen der Tubuli gerichteten Fettkörnchenströmung.

Abgesehen von einer Bestätigung der mir aus der Litteratur bekannten Thatsache, dass alle bisher daraufhin untersuchten Hoden fetthaltige interstitielle Zellen besitzen, fand ich in meinen vergleichend-anatomischen Untersuchungen eine Reihe von Anhaltspunkten nicht nur für die Richtungsbestimmnng der Fettkörnchenströmung, sondern auch für die Erkenntniss der Bedeutung dieser Strömungen für die Physiologie der Spermatogenese. Ich möchte an dieser Stelle auf eine eingehende Darstellung dieser Verhältnisse verzichten, und nur ein Objekt zur Vergleichung heranziehen, den Mäusehoden. - Ich fixirte die Drïse in toto olne Einstich in $\mathrm{Her}$ ann'scher Flüssigkeit, behandelte sie dann weiter wie den Katerhoden, ohne jedoch die Albuginea abzuziehen, und zerlegte sie schliesslich in Serienschnitte von $5-10 \mu$ Dicke. Das Bild ist ein wesentlich anderes, als das des Katerhodens!

Wie Figur 3 zeigt, ist die Zwischensubstanz hier nur in ganz geringem Grade ausgebildet, indem in den von den Tubuli freigelassenen rautenförmigen und von Bogenlinien begrenten Räumen nur einige ein bedeutend feiner vertheiltes Fett enthaltenden interstitiellen Zellen anzutreffen sind. Im übrigen legen sich die abgeplatteten Wandungen und Windungen der Tubuli unmittelbar aneinander an. Dagegen fällt uns sofort sowohl die im ganzen stärkere Osmiumtinktion des Epithels, als anch eine in dem meisten Tubuli vorhandene ziemlich dichte Randzone schwarzer Fettkörnchen auf. Diese im Inneren der Tubuli gelegenen Fettkörner sind ron einer Reihe von Autoren zum Gegenstande eingehender Untersuchungen gemacht worden.

B rown (bei Ebner) hebt hervor, dass zu der Zeit, wo die jungen Spermatozoen sich mit den Sertolischen Zellen ver- 
binden, um die Kerne der letzteren grosse Kugeln zu finden seien, die sich mit Osmium schwarz fürben, und glanbt, dass diese Kugeln z. Th. den Zweck der Ernährung einer neuen Spermatozoengruppe liaben, wenn auch die stützende Funktion der Sertolischen Zelle die Hauptsache sei.

$\mathrm{Eb}$ er (15) beschreibt in seinen Spermatoblasten dann eine Fettkörnchenströmung, deren verschiedene Stadien stets mit ganz bestimmten Stadien der Spermatogenese zusarnmentreffen. Es war mir leider nicht möglich, die ganze Litteratur der letzten acht Jahlre über die Spermatogenese zu berücksichtigen und ich muss mich bezüglich der Fettkörnchenströmung mit einer Kritik der E b n e r'schen Anschaungen begnügen.

Ebner beginnt seine sich auf den Rattehoden beziehende Beschreibung folgendermaassen: Zur Zeit der Reifung der Samenfäden sieht man die Protoplasmalappen, welche jedem derselben anhängen, ein immer stärker körniges Ausselien gewinnen, und an Präparaten aus Flemming's Gemisch bräunen sich diese Lappen erst mehr diffus, später aber färben sich deutlich einzelne Körnchen scliwarz, welche erst zahlreich und klein, spüter aber weniger zahlreich, aber grösser und tiefschwarz erscheinen. Ebner findet die Fetttropfen dann zunächst zwischen den Köpfen der abgestossenen Samenfäden, später in einer Zone zwischen den ins Centrum gelangten Spermatozoen und den nach aussen von ihnen liegenden Samenzellen. In diesem Stadium sollen die Fussplatten der Sertolischen Zellen vollständig frei von Fett, und "keine einzige durch Osmium 'geschwärzte Kugel" in denselben zu bemerken sein. Darauf lässt Ebner die Fettkörnchen von dem Centrum der Tubuli in den Fuss der Sertolischen Zellen wandern und von dort aus wieder in gelöstem Zustande die Wanderung in die Protoplasmalappen beginnen.

Ebner geht bei dem Studium dieser Fettkörnchenströmung offenbar von einem nicht gut gewählten Punkte ans, und die Folge davon ist, dass er sich am Schlusse seiner Betrachtung in Verlegenheit sieht bei der Beantwortung der allerdings sehr berechtigten Frage: Wie kommen denn bei der Bildung der ersten Spermatozoen die Fettropfen in die Protoplasmalappen?

Wenn man nun nicht die meiner Ansicht nach absurde Annahme machen will, dass die in den Sertolischen Zellen offenbar nach ganz bestimmten Gesetzen strömenden Körner ganz 
zufällige, bedeutungslose Gebilde sind, so bleiben nur zwei Möglichkeiten übrig. Entweder sie stellen bei der Spermatogenese nicht verbrauchtes Material dar, welches auf dem gewöhnlichen Wege der Ernährung der Gewebe in die Protoplasmalappen grelangt und nun dort als überflüssig ausgeschieden wird, oder aber es repräsentirt einen für die Bildung der Samenfäden unentbehrlichen Nührstoff, der ganz oder theilweise verbraucht wird. Wenn nun die Körnchenströmung wirklich nur zwischen der Fussplatte der Sertolischen Zelle und dem Centrum der Tubuli als Grenzpunkten sich abspielte, so müsste bei Annahme der ersten Möglichlseit alsbald eine kolossale Anhäufung unverbrauchter Stoffe in den Fussplatten eintreten, während sich bei Annahme der zweiten Möglichkeit ebenso bald gänzlicher Mangel des notwendigen Nährstoffes einstellen würde.

Ebner sieht nun in dem. Fette einen Nährstoff für die Spermatozoen und kommt naturgemäss zu dem Schlusse, dass die Ergänzung des verbrauchten Materials in den Fussplatten der Sertolischen Zellen stattfinden müsse. Bestärkt wird er darin noch durch die Beobachtung, dass die kurz nach der Bildung. der Spermatoblasten in den Fussplatten auftretenden Fettkörner bedeutend grösser sind, als die nach der Reifung der vorigen Spermatozoengeneration in das Innere des Tubulus abgestossenen.

Ich theile den Standpunkt Ebner's und dies um so eher, als ich in $\mathrm{Her} \mathbf{m a n} \mathbf{n}$ 'schen Präparaten des Hundehodens eigenthümliche, in der Axe der reifenden Spermatozoen gelegene, mit einem durch Osmium geschwärzten Fettmantel versehene Hohlcylinder antraf. (Fig. 8).

Im grossen und ganzen kann ich auch die Schilderung Ebner's von der Körnchenströmung bei meinen Präparaten von der Maus bestätigen, und möchte mich nur bezïglich der fettresorbirenden Funktion der Fussplatten auf einen extremeren Standpunkt stellen. Wenn ich auch nicht leugnen kann, dass Bilder, wie $\mathrm{Eb} \mathrm{ner}$ sie beschreibt, vorkommen, in denen sämmtliches Fett in dem Lumen der Tubuli angetroffen wird, und "auch nicht eine einzige durch Osmium geschwärzte Kugel" in den Füssen der Spermatoblasten zu bemerken ist, so finde ich doch nicht selten Stellen, wo von einer erfolgten Riückwanderung des Fettes noch nicht die Rede sein kann, und sich trotzdem 
schon eine Randzone scbwarzer Tropfen neu gebildet hat. Später erst setzt die Rückwanderung des central gelegenen Fettes ein, welcher Vorgang gar keine Aelinlichkeit mit der central gerichteten Fettströmung hat. Was diese letztere anbetrifft, so glaube ich nicltt, dass sich bei ihr das Fett in absolut gelöstem Zustande befindet, vielmehr finde ich in ganz frischen Präparaten an Stellen, welche der direkten Einwirkung des Osmiums ausgesetzt waren, allerfeinste Körnchen zu zarten Reihen angeordnet, welche das in der Fussplatte gelegene Fett mit dem in den Protoplasmalappen bereits ausgeschiedenen verbinden.

Während ich es im Gegensatze zu Ebner für bewiesen erachte, dass in Wirklichkeit ein unverbranchter Theil des anfangs in der Fussplatte gelegenen Fettes in den Protoplasmalappen ausgeschieden wird, vermisse ich also jeden beweisenden Umistand, dass das in die Protoplasmalappen einmal ausgeschiedene Fett nach seiner Rückwanderung denselben Weg noch einmal nimmt.

Um mich nicht zu sehr von meinem eigentlichen Gegenstande zu entfernen, möchte ich hier, soweit als müglich unter Vermeidung theoretischer Erörterungen, meine Auffassung von den Fettkörnchenströmungen in den Sertolischen Zellen der Maus präcisiren, and ich wähle mir zum gedachten Ausgangspunkt die Verhältnisse bei der Bildung der ersten Spermatozoenpruppe überhaupt, wie dieselben auf Grund der Erscheinungen bei den späteren Produktionen mit logiseher Nothwendigkeit vorausgesetzt werden müssen.

Sobald die Kerne der ersten Spermatiden das Centrum ihrer Zellen zu verlassen beginnen, sammeln sich "in dem Fusse der Sertolischen Zelle zahlreiche kleinste Fettkörnchen an (cf. Fig. 6c), welche konfluiren und melir oder weniger grosse Kugeln und unregelmässig gestaltete Körner bilden. Diese Körner geben schon bald feinste Theile ihrer Substanz an das Protoplasma der Sertolischen Zellen ab, und die Kerne der Spermatiden haben kaum eine excentrische Lage eingenommen, als auch schon in den protoplasmatischen Theilen derselben die ersten feinen Fetttröpfchen auftreten, die übrigens auch in der ganzen Länge der jetzt sehr schmalen Sertolischen Zelle angetroffen werden. Sind nun die Spermatidenkerne ganz in das peripherische Ende ihrer nunmehr gestreckten Zellen gerlickt und haben bereits eine längliche und 
zugespitzte Form erhalten, so scheinen zwar die Tröpfchen in den Protoplasmalappen verschwunden za sein, in Wirklichkeit sind sie nur von der mittlerweile eingetretenen diffusen Briuntung derselben verdeckt und lassen sich bei genauem Zusehen noch nachweisen. Die Fettrropfen in den Fussplatten zeigen in diesem Stadium kaum eine wesentliche Verminderung ilurer Substanz. Sobald nun die Bildung der nächsten Spermatidengruppe beginnt (Fig. 6a), finden wir eine nicht unbeträchtliche Ansammlung von Fett in den Fussplatten, und nun können wir Schritt für Schritt verfolgen, wie die Körnchen in den Protoplasmalappen erst deutlicher, dann grösser werden, schliesslich sich einander nähern und aus 10-20 Körnchen bestehende äusserst zierliche Rosetten bilden (Fig. 6b). Parallel hiermit konstatiren wir das allmähliche Verschwinden des Fettes aus den Fussplatten und ein Vorrticken der reifenden Spermatozoen gegen die Peripherie des Tubulus. Indem nun neue Spermatogonien ihrer Theilung entgegen wachsen und der ganze Epithelbelag höher wird, gelangen - sei es durch aktive, sei es durch passive Bewegungen - die Sperma. tozoen in das Lumen der Tubuli, und wir finden dort zwischen ihren Köpfen eigenthümliche, durch Osmium schwach gebräunte und einige tiefschwarze Fettkörnchen enthaltende Kugeln von etwas geringerer Grösse als die Spermatidenkerne. Wir erkennen in diesen Kugeln sofort die veränderten Protoplasmalappen der früheren Stadien (Fig. 6c). Wïhrend nun kurz vorher alles Fett aus den Fussplatten verschwunden war, sammeln sich dort um diese Zeit in der Regel schon wieder einige Fetttropfen an, ohne dass irgend eine Beziehung derselben zu den im Centrum der Tubuli gelegenen matten Kugeln mit ihrem Inhalte an Fett zu erkennen wäre. Die Rückwanderung dieser Kugeln beginnt etwas später und ich möchte in derselben überhaupt einen Voryang von geringerer Bedeutung sehen. Jedenfalls wäre die Annahme, dass diese Kugeln in die abführenden Lymphwege ausgeschieden werden, viel rationeller, als die ebenso wenig bewiesene Behauptung Ebner's, dass die in das Lumen ausgeschiedenen Fettkörner den Weg durch die Sertolische Zelle in derselben Richtung zum zweiten Male machen. Auch die Thatsache dass sich im Nebenhoden sowohl wie im vas deferens mit feinsten Fettkörnchen angefüllte Kageln finden, die hier vielleicht auch nährende Funktion haben, scheint mir eher dafür als da- 
gegen zu sprechen, dass die rückwandernden Fetttropfen aus unbranchbarem Material bestehen. Und schliesslich clarf Ebner nicht vergessen, dass wohl Fett, nicht aber solche Protoplasmakugeln in den Sertolischen Zellen gegen das Lumen zuströmen. Bevor er also die Fetttropfen die Reise zum zweiten Male machen lïsst, muss er sie von den Protoplasmakugeln trennen, mit welchen sie sehr eng verbunden zu sein scheinen. Ich will hier die sich mir aufdrängende Frage nicht weiter verfolgen, warum sich lenn überhaupt die reifenden Spermatozoen mit einem Ueberschuss an brauchbarem Material beladen könnten, der später unverbrancht wieder abgeschieden werden muss, sondern nur bemerken, dass ich aus Gründen, welche aus der oben gegebenen Schilderung (ler Fettkörnchenströmung klar ersichtlich sind, in der von Brown und Ebner den Sertolischen Zellen mehr als neben. sïchlich zugestandenen Funktion der Resorption von geformtem Fett aus dem nährenden Gewebssafte, gerade die specifische und im höchsten Grade wesentliche Funktion dieser Zellen sehe. Für mich hat die Aunahme am meisten Wahrscheinlichkeit, dass sämmtliches zur Ernährung einer jeden reifenden Spermatozoengruppe nothwendige Fett in jedem einzelnen Falle von der Sertolischen Zelle - und zwar nicht im Ueberschusse - resorbirt, und ein nicht brauchbarer Bestandtheil desselben, von anderer chemischer Constitution, in das Lumen des Tubulus ausgeschieden und mitsammt anderen Verbrauchsprodukten gclöst in die abführenden Lymphwege ausgeschieden wird. Ein geringer Rest brauchbaren Materials begleitet den Samen auf seinem weiten Wege durch Nebenhode und vas deferens.

Die zu Gunsten der Deutlichkeit etwas schematisierten Figure 6a-c stellen einige wichtige Stadien der Spermatogenese bei der Maus mit den zugehörigen Phasen der Fettkörnchenströmungen dar, wie sie aus einer grösseren Anzahl beobachter Stellen als die Regel erkannt worden sind. Die Erklärung der Zeichnungen ist in den vorstehenden Anseinandersetzungen enthalten, und ich möchte hier nur nochmals darauf aufmerksam machen, dass zu einer Zeit, wo die Spermatidenkerne noch kaum den Rand ihrer Zellen erreicht haben, sowie kurz vor der Theilung. der Spermatogonien eine besonders starke Resorption von Fett in den Fussplatten stattfindet oder eben stattgefunden hat.

Kehren wir nun nach dieser Abschweifung wieder zum 
Katerhoden, dem eigentlichen Gegenstande unserer Betrachtungen zurück, so finden wir hier eine ähnliche Fettkörnchenströmung. Wie Figur 5a zeigt, sehen wir auch bier in den protoplasmatischen Theilen der Spermatiden, deren Kerne kaum noch gegen den Rand der Zellen gerückt sind, eine äusserst zierliche Vertheilung feinster Fettkörnchen. In einem späteren Stadium sind dieselben wie beim Mäusehoden durch eine dunklere diffuse Färbung der Protoplasmalappen verdeckt (Fig. 5b), um schliesslich (Fig. 5c) bei der Abstossung der reifen Spermatozoen mit diesen in das Lumen der Tubuli ausgeschieden $z \mathfrak{u}$ werden. Während wir aber beim Mäusehoden ein diesen Vorgängen parallel gehendes Verschwinden von Fett in der Randzone verfolgen können, fehlt, wie schon früher hervorgehoben, diese Randzone beim Kater vollständig, und die einzige Anhäufung grösserer Fettmengen finden wir in den hier ungleich mächtiger als bei der Maus entwickelten interstitiellen Zellen.

Legt uns nun schon die vergleichende Erkenntniss dieser Thatsachen die Vermuthnng sehr nahe, dass wie bei der Maus das in das Lumen ausgeschiedene Fett aus der Randzone stammt, so analoger Weise die im Inneren der Tubuli beim Katerhoden liegenden Fettröpfchen in ihrem Ursprunge auf das Fett der interstitiellen Zellen zurückzuführen sind, so wird diese Vermuthung zu der in diesen Dingen überhaupt möglichen Gewissheit durch den Nachweis der oben bereits flüchtig beschriebenen Kanäle und einer durch dieselben stattfindenden Strömung von Fett.

Diese die Wandung der Tubuli durchbohrenden und die interstitiellen Zellen mit den Sertolischen verbindenden Kanäle treten nun keineswegs immer mit der Deutlichkeit in die Erscheinung, wie sie in Fig. 4a und 5e genau nach der Natur dargestellt sind. Sie werden überhaupt nur selten in ibrer ganzen Länge angetroffen in Schnitten, welche senkrecht zur Längsaxe der Tubuli geführt sind, worans ich den Schluss ziehe, dass sie in der Regel mit ihrem Inhalte an Fett keine direkte Fortsetzung der Sertolischen Zellen bilden, sondern zu der Längsaxe derselben in einem stumpfen Winkel geneigt sind. Am leichtesten finden wir die Kanälchen in den Stadien der Spermatogenese, in welchen wir bei der Maus die grössten Ansammlungen von Fett in den Fussplatten konstatierten. Sie sind alsdann meistens mit 
Fett gefüllt und fallen uns an Stellen, welche schrïg zur Axe der Tubuli geschnitten sind, als bald mehr, bald weniger zugespitzte, längliche, mit helleren Contouren versehene, mehr oder minder dunkel gefürbte Bälkchen auf, welche die zu beiden Seiten scharfen Contouren der Wandungen unterbrechen. In der Figur 7 habe ich eine Anzahl derartiger Kanälchen wiedergegeben, welche wohl keines weiteren Commentares bedürfen.

Ich legte mir nun die Frage vor: Entstehen diese Kanälchen in jedem einzelnen Falle der Durchwanderung eines Fetttropfens, oder sind es prïformirte Gebilde, und wann treten dieselben zum ersten Male auf?

Während ich diese letzte Frage völlig: unentschieden lassen muss, möchte ich mich doch für die präformirte Natur dieser Gebilde entscheiden. Zunächst bestimmen mich dazı Befunde, wie sie Fig. 4 a und 5 c zeigen. Wenn sich die Kanälchen erst unter dem Drucke des durch irgend welche chemischen oder mechanischen Momente in Bewegung gesetzten Fettkörnchens in jedem einzelnen Falle bilden sollten, so nuïssten sie sich meiner Ansicht nach auch sofort wieder zuziehen, sobald das Fettkörnchen in das Innere des Tubulus gelangt ist. Fig. $4 \mathrm{a}$ und $5 \mathrm{c}$ jedoch zeigen das Gegentheil. Zweitens aber finde ich auch an Stellen, welche vollständig frei von Fett sind, Lücken in der Wandung der Tubuli, welche mit den fetthaltigen Kanälchen grosse Aehnlichkeit haben.

Schliesslich fallen, wenn anch in geringem Grade, eigenthïmlich geschlängelte Substanzdefekte ins Gewicht, welche ich - gewiss nicht als der erste - des öfteren in den Fussplatten der Sertolischen Zellen fand, und die ich in einigen Fällen durch die Wandung der Tubuli hindurch in die interstitiellen Räume hinein verfolgen konnte, ohne dass sich in unmittelbarer Nähe Fett befunden hätte.

Einmal auf diese Verhältnisse beim Katerhoden aufnerksam geworden, glaube ich, trotz der äussersten Feinheit der Strukturen, auch beim Mäusehoden Stellen gefunden zu haben, welche mir einen Uebertritt von geformtem Fett aus den interstitiellen Zellen in die Tubuli zu beweisen scheinen.

Während wir dies beim Kater als die Regel bezeichnen können, müsșen wir die Fetttröpfchenbildung bei der Maus in die Fiisse der Sertolischen Zellen verlegen, und hiermit scheint mir 
das bei der Maus viel stärkere hervortreten der Fusskerne in Einklang za stehen. Ueberhaupt kann ich mich des Gedankens nicht erwehren, dass wir in den Fusskernen und den.Kernen der interstitiellen Zellen funktionell verwandte Gebilde zu sehen haben. Oder sollte z. B. beim Kater nicht nur das Fett, sondern die ganze Zelle mit dem Kerne in das Innere der Tubuli wandern? Dafür könnte allenfalls Fig. 4 sprechen, in welcher wir im äusseren Ende des Kanälchens ein gegen das Lumen zugespitztes, abgeschnittenes Stück eines Kernes sehen, sowie die merkwürdigen Form- und Lageveränderungen der Fusskerne, welche, nach den von vielen Autoren gegebenen Abbildungen, zuweilen aus der Wand der Tubuli gleichsam herauszuwachsen scheinen. Mehr als eine Anregung zu einer Prüfung dieser Fragen soll in diesen Zeilen nicht liegen. Für den Katerhoden möchte ich eine Wanderung der Zellen für unwahrscheinlich halten, da die Kanäle in der Regel hierfür zu klein sind und ich in der funktionirenden Drüse eine die auswandernden Zellen ergänzende Neu-Entstehung: aus fixen Bindegrewebszellen, wie ich sie für den sich entwickelnden Hoden demnächst zu beschreiben gedenke, kaum nachweisen kann, und Mitosen niemals zur Beobachtung kamen. Allerdings will ich die Möglichkeit einer direkten Kerntheilung nicht ohne weiteres von der Hand weisen. Zellen mit 2 Kernen finden sich nicht allzu selten.

Wenn wir nun zum Schlusse den unsern vorliegenden Anseinandersetzungen zu Grunde liegenden Gedankengang kurz zusammenfassen, so sahen wir uns durch merkwürdige Befunde von Kanälchen, welche zu Fettströmungen im Katerhoden Beziehungen zu haben schienen, veranlasst, uns über die Bedeutung des Fettes für die Spermatogenese überhaupt zu orientiren. Auf Grund der Befunde beim Mäusehoden, kamen wir dann in Uebereinstimmung mit der Ansicht nambafter Autoren zu der Ueberzeugung, dass das Fett ein Nährmaterial für die reifenden Spermatozoen darstelle und $\mathrm{zu}$ diesem Zwecke in bestimmten Stadien der Spermatogenese in einer von der Wand der Tubuli gegen das Lumen derselben gerichteten Strömung begriffen sei. Während wir aber bei der Maus eine im Inneren des Tubuli gelegene peripherische Fettzone als Ausgangspunkt dieser Strömung bezeichnen konnten, sahen wir uns bei dem Fehlen dieser Randzone im Katerhoden bei diesem Objekte genöthigt, die auch hier vorhandene Fettkörnchen- 
strömung in ihrem Ursprunge auf das im Gegensatz zu den Verhältnissen bei der Maus hier stark entwickelte interstitielle Fettlager zurtickzuführen. Hier nun wurden die in ihrer Aechtheit von uns selbst anfangs angezweifelten Kanäle zu einem theoretischen Postulate, und es gelang uns nun leicht, sie gerade in denjenigen Stadien der Spermatogenese beim Kater aufzufinden, in denen wir bei der Mans eine vermehrte Anhäufung von Fett in den Fussplatten fanden.

Diese Art der Darstellung entspricht anch dem thatsächlichen Gange meiner Untersuchungen.

Während also frühere Autoren sich mit der Beschreibung der interstitiellen Substanz begnügten, oder sich hinsichtlich ihrer Bedeutung in Vermuthungen ergingen, die auf der breiten Basis anatomischer und physiologischer Möglichkeiten ruhten, glaube ich in den interstitiellen Zellen, des Katerhodens wenigstens, ein Organ sehen zu dürfen, welches das für die Ernührung der reifenden Samenfäden nöthige Fett resorbirt und aufspeichert, und halte für den wichtigsten Befund die beschriebenen Kanäle, welche die aufgespeicherten Vorräthe dem Orte ibrer Bestimmung entg'egenfiühren.

Wenn wir nun in den interstitiellen Zellen überhaupt ein für die Spcrmatogenese wichtiges trophisches Hïlfsorgan sehen müssen, worüber ja erst weitere Untersuchungen die Entscheidung bringen könmen, inwieweit sind dann die in der Literatur zerstreuten Angaben in diesem Sinne zu verwerthen?

Anf jeden Fall spricht Hofmei ster's (כ) Angabe von einer Vermehrung der interstitiellen Substanz im funktionirenden Hoden gegenüber dem ruhenden, für unsere Auffassung. Es spricht ferner dafür die interessante Beobachtung $\mathrm{H}$ ansemann's beim Murmel. thier (l. c.). Im übrigen jedoch müssen wir alle älteren Beobachtungen mit äusserster Vorsicht aufnehmen, da auf die physiologische Veränderlichkeit des Organs bisher zu wenig Rücksicht genommen wurde and vor allem die Begriffe einer Vermehrung oder Verminderung der Zwischensubstanz sehr relative sind, indem zum Beispiel eine Vermehrung derselben sowohl anf einen noch mangelhaften Entwicklungsgrad der Tubuli in embryonalenund Jugendzuständen des Individuums, als auch auf eine wirkliche Vermehrung der Elemente unter Volumzunahme des ganzen Hodens und schliesslich anf eine Rückbildung der Tubuli bei 
pathologischen und vielleicht auch senilen Zuständen zurückgeführt werden kann. Nicht einmal über einen so wichtigen Punkt, wie die Betheiligung der Zwischensubstanz an dem Aufbau des normalen, funktionirenden Menschenhodens herrscht Uebereinstimmung unter den Autoren. Hansemann findet die Substanz beim 24 Jahre alten Manne kaum mehr auf, während Reinke sie - in Uebereinstimmung mit Henle - bei einem 25jährigen Manne in mächtiger Entwickelung sieht. Doch darüber in einer folgenden Mittheilung mehr.

Es ist mir ein Bedürfniss, dem Direktor des Instituts, Herrn Prof. O. Hertwig, für das meiner Arbeit entgegengebrachte Interesse und die Förderung meiner Bestrebungen, sowie Herrn Privatdozenten Dr. Rudolf Krause für die Liebenswürdigkeit seines Rathes in allen Fragen der Technik, und seiner Hülfe bei einer nicht geringen Anzahl von Versuchen, auch an dieser Stelle meinen herzlichen Dank abzustatten.

\section{Literatur-Verzeichniss.}

1. Kölliker, Mikroskopische Anatomie oder Gewebelehre des Menschen. Bd. II. Leipzig 1854.

2. Henle, Handbuch der systematischen Anatomie 2. Aufl. Bd. II. Braunschweig.

3. Leydig, Lehrbuch der Histologie des Menschen und der Thiere. Frankfurt a. M. 1857.

4. Ludwig u. Tomsa, Die Lymphwege des Hodens. Sitzungsber. d. math.-naturw. Classe der k. Acad. der Wissenschaften. Bd. XLVI. Wien 1862.

5. Stricker's Handbuch der Gewebelehre. Leipzig 1871.

6. Hofmeister, Untersuchungen über die Zwischensubstanz im Hoden der Sïugethiere. Sitzungsbericht d. math.-naturw. Klasse d. k. Akademie d. Wissensch. Bd. LXV. Abth. III. Wien 1872.

7. Mihalkowicz, Beiträge zur Anatomie und Histologie des Hodens. Arbeiten aus d. phys. Anstalt zu Leipzig. Herausgegeb. v. Ludwig. Leipzig 1874.

8. Harwey, Ueber die Zwischensubstanz der Hoden. Centralblatt f. d. med. Wissenschaft. 1875. Nr. 30 .

9. Waldeyer, Ueber Bindegewebszellen. Arch. f. mikr. Anat. Bd. 11.

10. Derselbe, Sitzungsberichte der Berliner Akademie. Sitzung vom 11. Juli 1895. 
11. A. Jacobson, Zur path. Histologie der traumatischen Hodenentzündung. Virch. Arch. Bd. 75.

12. W. Nagel, Ueber die Entwicklung des Urogenitalsysterns heirn Menschell. Arch. f. mikr. Auat. Bd. 34.

13. D. Hansemann, Ueber die sog. Zwischenzellen des Hodens und deren Bedeutung bei path. Veriinderungen. Virch. Arch. 1895. Bd. 142.

14. Fr. Reinke, Beitriige zur Histologie des Menschen. Arch. f. mikr. Anat. Bd. XXXXVII. 1896.

15. v. Ebner, Zur Spermatogenese bei den Sïugethieren. Arch. f. mikr. Anat. Bd. 31. 1888.

\section{Erklärung der Abbildungen auf' Tafel XII.}

Siimmtliche Abbildungen sind in ihren Unrissen mit Hülfe des Abbe'schen Zeichenapparates in der Hölse des Objekttisches entworfen. Fig. 1-3 sind gezeichnet mit Zoiss DD und Ocular 2 , und darauf um die Hälfte verkleinert worden. Fig. $4-8$ sind mit Zeiss. Homog. Immersion $1 / 12$ und Ocul. 2 gezeichnet und in natürlicher Grösse wieder gegeben.

Fig. 1. Schnitt durch den Hoden eines Katers. Die dunklen Partien stellen die interstitielle Substanz dar. Hermann.

Fig. 2. Schnitt durch den Hoden eines Katers. Der Epithelbelag ist fortgelassen. Zenker-Heidenhain. - Die grossen interstitiellen Zellen.

Fig. 3. Hoden der Maus. Hermann. Schwach entwickelte interst. Substanz, dagegen Fettrandzone.

Fig. 4 a. Katerhoden. Hermann. Bei $K$ grosses Kanalchen mit keilförmigem Fettliorn.

Fig. 4 b. Nächster Schnitt der Serie, Reste des Fettkorns.

Fig. 5a-c. Katerhoden. Hermann. Die verschiedenen Stadien der Fettkörnchenströmungen beim Kater. Kanälchen in der Wandung der Tubuli.

Fig. 6a-c. Mäusehoden. Hermann. Die verschiedenen Stadien der Fettkörnchenströmungen bei der Maus.

Fig. 7. Einige Kanälchen ohne den zugehörigen Epithelbelag.

Fig. 8. Spermatozoen vom Hund mit Fetteylindern. 